\title{
Treatment of malunion in ankle fractures
}

\author{
D. Weber $\cdot$ N. Borisch $\cdot$ M. Weber
}

Received: 12 August 2010/ Accepted: 2 November 2010/Published online: 24 November 2010

(C) Urban \& Vogel 2010

\begin{abstract}
Malunions are fractured bones that have healed in pathological positions. This leads to nonphysiological load transfer. Clinical symptoms at the ankle may include swelling, pain and impaired function. Lateral, posterolateral or posteromedial subluxation of the talus will be visible on the radiographs. Surgical correction may be indicated if the malunion is symptomatic. Different osteotomies have been described, but the goal of surgery will always be the reduction and retention of the subluxed talus in an effort to recreate stable conditions. Eighty percent of patients show good results with significant pain reduction in mid-term follow-up studies. Ankle arthrodesis after corrective osteotomy is rarely necessary.
\end{abstract}

Keywords Ankle · Fracture - Malunion - Malleolus · Osteotomy

\section{Introduction}

A malunion is a fractured bone that has healed in a nonanatomical position. Malunions can occur in diaphyseal and articular fractures. Pathological shortening, angulation

\section{Weber $(\bowtie)$}

Department of Surgery, Clinic for Orthopaedics and

Traumatology, St. Clara Hospital, Kleinriehenstrasse 30, 4018 Basel, Switzerland

e-mail: daniel.weber@claraspital.ch

\section{N. Borisch}

Clinic for Orthopaedics and Handsurgery, DRK-Klinik, Baden-Baden, Germany

M. Weber

Department of Orthopaedic surgery, University Hospital Insel, Bern, Switzerland and/or rotation can be present where there is malunion. Irregularities of the joint surface are present in malunions of articular fractures. All these conditions lead to abnormal weight transfer and can cause arthritic joint destruction.

Clinically, malunions lead to joint instability, which can be controlled by good muscle function in some cases. In more severe deformations, arthritic changes and/or poor muscle quality can cause pain, swelling and impairment of function. These conditions are indications for surgical correction of malunion. When considering surgery, the patient's age and occupation as well as their range of motion should be taken into account [1]. The duration of the malunion is not the most important factor. The most important indication for surgery is pain. If no or few degenerative changes are present, an osteotomy of some sort should be performed [2-4]. Only in cases of severe arthritis and/or deformation will ankle arthrodesis be indicated [5-8].

Malunions are caused by inappropriate surgical techniques, misjudged fracture instability and therefore insufficient fixation, inaccurate reduction, and/or an immobilization time that is ineffective or too short. This will apply equally to osteosynthesis and nonsurgical treatment.

The pathological anatomy of malunion is defined by the initial trauma and the lesions that have occurred. This means that the initial morphology of the instability caused by the fractures and ligamentous lesions will also be found in the malunion (in the case of an untreated fracture). Unstable fractures may be treated in different ways using a multitude of implants. If all fractures that could render the ankle unstable (i.e., malleolus medialis and lateralis, Volkmann's triangle, posteromedial corner, impaction of the tibial pilon, or combinations of these) are treated by open reduction and internal fixation, the joint should be stabilized and in situ healing should occur. If an unstable ankle fracture is combined with insufficient or partial 
osteosynthesis, malunion can be expected after bone healing.

It may be difficult to judge the extent to which any part of a complex fracture contributes to an unstable joint. Generally, the position/translation of the talus will help to explain the lesions:

- If there is lateral translation, we will find lesions of the deltoid ligament or the medial malleolus and fracture of the lateral malleolus (Fig. 1)

- If there is posterolateral translation, we will find a ruptured deltoid ligament and fractures of the lateral malleolus and Volkmann's triangle [9]

- If there is posteromedial translation, we will find fracture of the lateral malleolus, rupture of the anterior tibiofibular ligament, and fracture of the posteromedial corner [10]

Of course, other fracture patterns can occur that cause similar conditions.

\section{Materials and methods}

\section{Radiological evaluation}

Standard or conventional radiographs are essential for the evaluation. Two projections are needed: anteroposterior and lateral views, both standing. It is essential to the evaluation of instability that these views be performed under load. This procedure will reveal the subluxation of the talus (lateral, medial, posterolateral, or posteromedial).

If there is any doubt about the extent of the posterolateral or posteromedial fracture/corner or the position of the malunited malleolus, a computer tomography is advocated. We believe that a CTscan with 3D-image reconstruction almost always yields important additional information
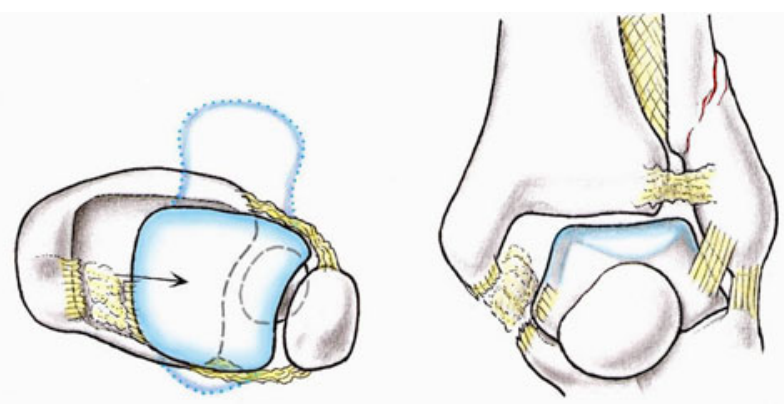

Fig. 1 Drawing of the left ankle (left view from below; right anterior view): rupture of the deltoid ligament, widened joint space, distortion/ rupture of the anterior tibiofibular ligament, fracture of the lateral malleolus with shortening and external rotation, translation of the talus about the pathological morphology of the bony structures (Fig. 2).

\section{Treatment}

After the malunion has been completely documented, a detailed treatment plan has to be drawn up. The plan should address surgical exposure, the condition of the soft tissues, osteotomy, the degree of correction needed, osteosynthesis, implants, and the post-op regime (immobilization, weight bearing).

The goal of surgical treatment is to stabilize the ankle by restoring its native anatomy. In terms of stability, this means that the subluxed talus has to be reduced into its correct position. Therefore, all parts of the ankle that have deformities that will interfere with repositioning of the talus have to be corrected by osteotomy.

Operations and surgical approaches

Operations to correct malunions of the ankle are osteotomy of the lateral malleolus alone, or with the medial malleolus, Volkmann's triangle or the posteromedial corner. An (additional) supramalleolar tibial osteotomy (Fig. 3) may be required if the alignment of the pilon joint line has been changed (pilon impaction fracture) [11]. Arthrodesis of the ankle is indicated in cases of severe destruction of the joint.

The patient is positioned supine, in the lateral decubitus or prone according to the osteotomies that have to be performed.

The surgical approaches should take the previous skin incisions into account. Devascularization of the skin flaps must be avoided absolutely. It may be appropriate to plan corrective surgery in collaboration with a plastic surgeon. Technical details of osteotomies around the ankle joint have been published by different authors. Therefore, the
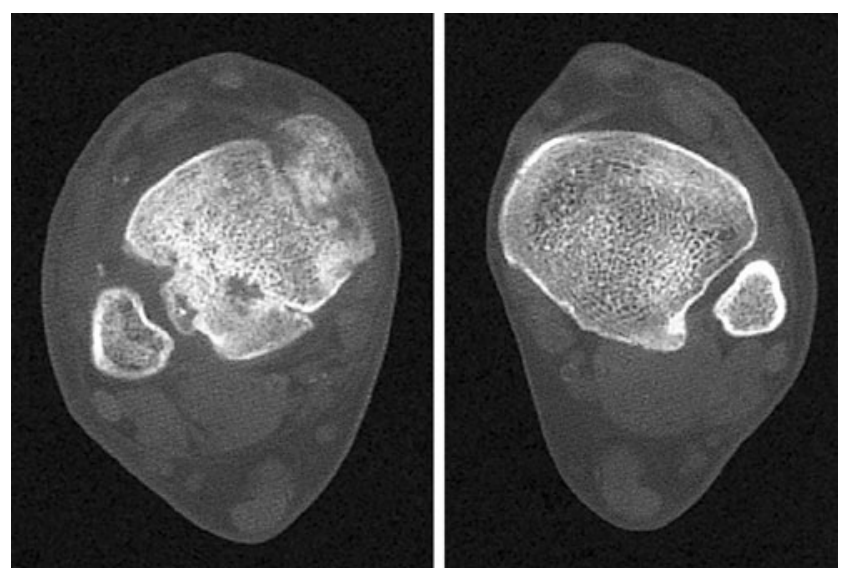

Fig. 2 CT scans of both ankles, showing the tibiofibular notch. Right normal. Left diastasis of the fibula and tibia, osteophytes on the tibial side 

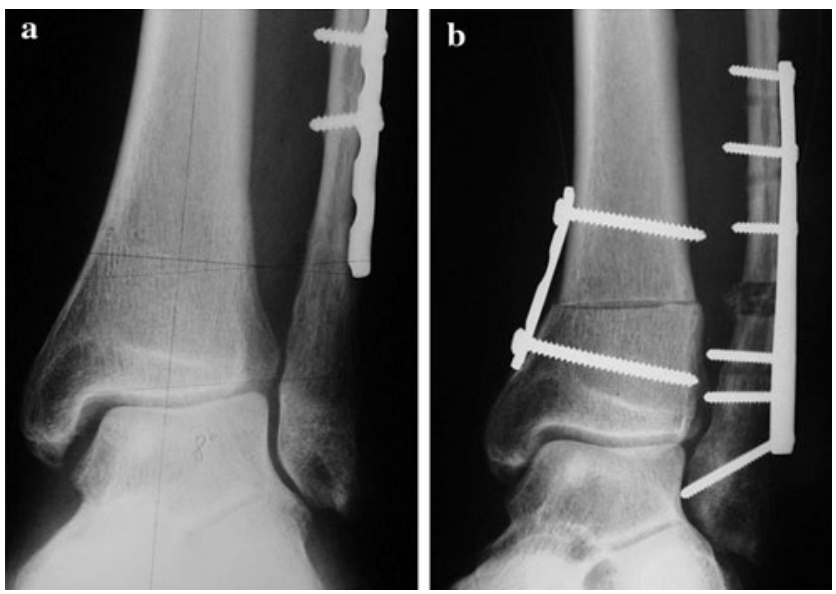

Fig. 3 a Radiograph of left ankle. Malunion after osteosynthesis of a high fibular fracture. Impaction fracture of the tibial pilon with valgus position. Talus subluxation. b Postop. radiograph of left ankle. Osteotomy and osteosynthesis of fibula and supramalleolar osteotomy. Talus reduced

only method we will describe in more detail here is the surgical approach to osteotomy of the fibula (Figs. 4, 5). For this lateral lengthening procedure, a transverse, oblique or z-shaped osteotomy of the lateral malleolus is performed. There are always osteophytes and scar tissue (Fig. 2) between the fibula and the tibia that have to be excised to allow distalization and derotation of the malleolus and repositioning of the talus. It may be necessary to free the widened joint space between the medial malleolus and the talus from any scar tissue that may have formed there before repositioning becomes possible. Then a 3.5 or $2.7 \mathrm{~mm} \mathrm{LC}-\mathrm{DCP}$ or LCP plate (AO-ASIF) is fixed to the lateral malleolus. The reduced position of the malleolus can be maintained by means of a tension/compression device, or simply by K-wires. A corticocancellous graft

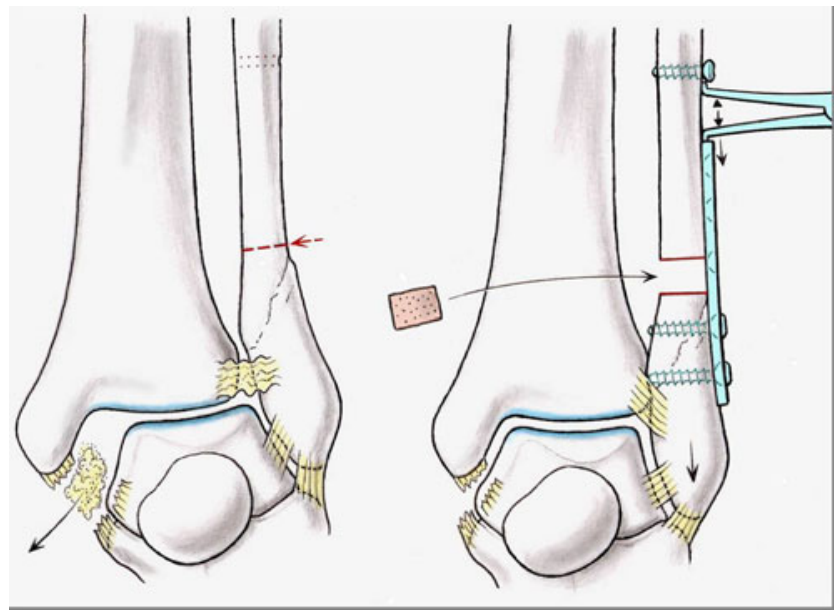

Fig. 4 Drawing of lateral malleolar lengthening osteotomy: removal of scar tissue between the medial malleolus and talus, osteotomy of the fibula, lengthening with distraction device, and grafting from the tibia, as initially described by BG Weber [8], is placed into the osteotomy gap; however, we believe that excised bone from the malunion does the job just as well, thus eliminating the need for additional trauma. Finally, the plate is fixed proximally. After irrigation of the wounds and closure, a plaster cast is applied. The ankle will be held in a neutral position for 6 weeks after the operation. Physical therapy may begin after wound healing. Weight bearing will not be allowed for the first 6 weeks.

\section{Results}

Radiologically, the reconstruction of the ankle mortise is documented in ap and lateral views. Bone healing can be expected after $8-12$ weeks. Clinically, $80 \%$ of patients show good results, achieve pain-free function with daily activities, and return to their pre-injury activity levels [2]. Functional outcome can be measured on the AOFAS score [1]. Progression of pre-existing degenerative signs in some patients is described in all series [2-8]. It can be estimated that, over a follow-up period of 3-7 years [2-4, 7], $10-15 \%$ of all patients will need further surgery in the form of ankle fusion.

\section{Discussion}

A malunion is a fracture that has healed in a pathological position. Since the introduction of guidelines and techniques for fracture reduction and fixation from the AOASIF, malunions have generally become rare. Longer life expectancy with more and more fractures in patients with osteoporotic bone (and diabetes mellitus) will possibly lead to a return of malunions, especially at the ankle. Indications for the surgical correction of malunions are impaired function and pain. Surgical principles and techniques are basically the same for initial fracture treatment and corrective osteotomies in patients with normal or porotic bone. It may be that newer plate designs with screws that have angular stability will allow safer fixation of the fracture fragments in some situations, especially in the presence of osteoporosis [12, 13].

The key issues in the treatment of ankle fractures and osteotomies are always the reduction and retention of the subluxed talus and the fixation of the fractured or osteotomized malleoli in their anatomical positions. Results after healed osteotomies are generally good, with $80 \%$ of patients reporting significant pain reduction and improved ankle function. The time between initial trauma and correction of the malunion in accordance with radiologically normal joint lines does not influence the outcome of the correction procedure $[2,7,8]$. However, it is logical to 

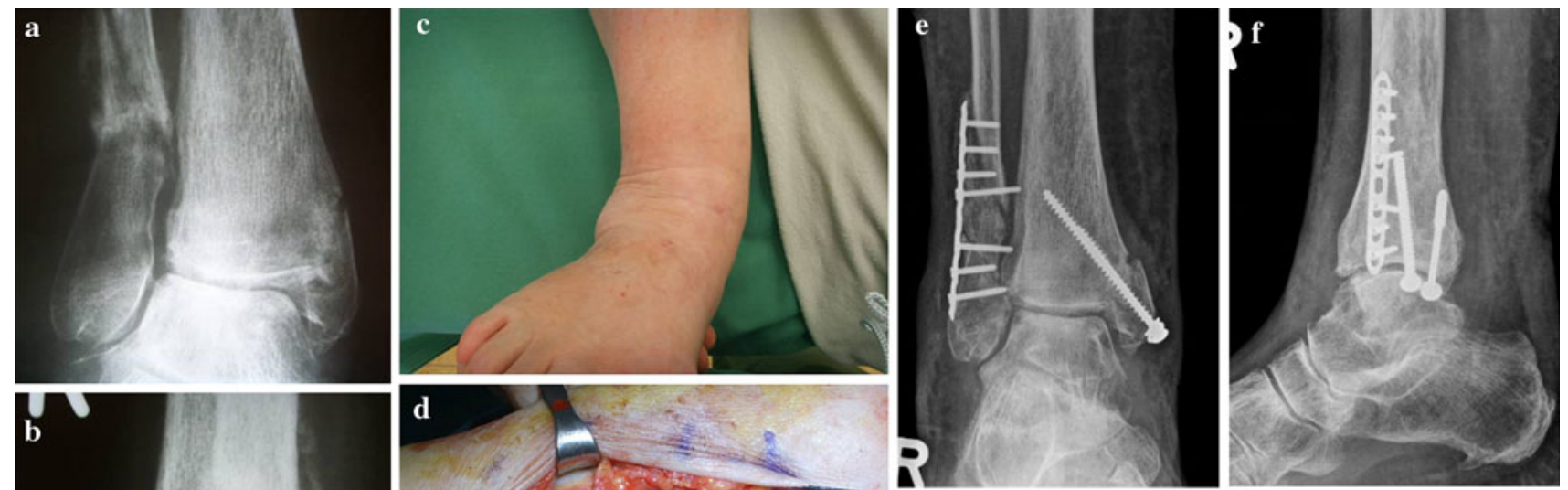
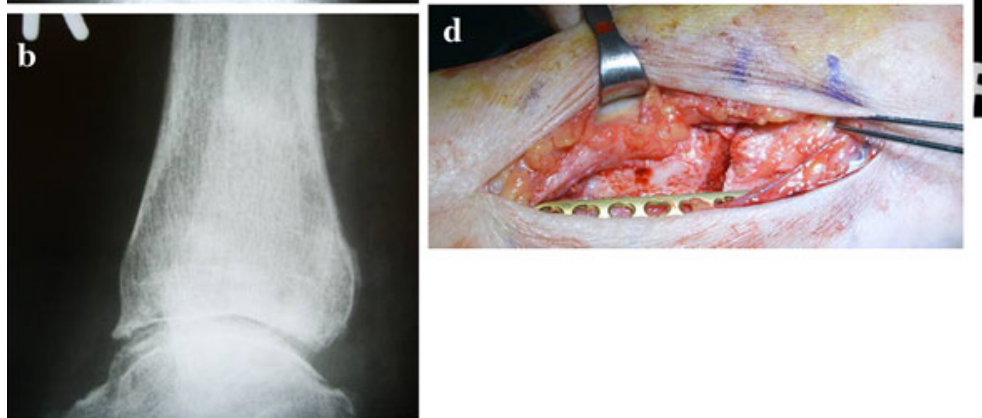

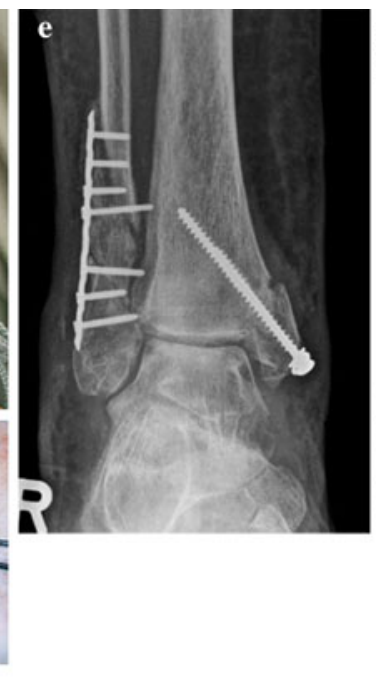

.
Fig. 5 a, b Radiographs (ap and lateral view) from the rehab hospital showing bimalleolar malunion in an 86-year-old female 3 months after falling out of bed after total knee replacement surgery. Treatment was strictly functional. Valgus position of the talus, widened joint space medially. c Clinical appearance. d Intraoperative

correct an unstable ankle as soon as possible, due to the problems associated with pathological load transfer at the malunited ankle. If degenerative changes are already present before the corrective osteotomy, they will not disappear after the restoration of normal axial alignment. Nevertheless, the progression of these arthritic changes will be slowed and the patients will benefit from substantial pain reduction. We believe that this is due to the re-balancing of the ankle and the normalization of load transfer. This not only reduces the stress on the cartilage and subchondral bone but also that on the soft tissues, which is probably more important for pain reduction.

Even if the degenerative changes do progress, a corrected and well-balanced ankle will respond more positively to an orthotic device. However, within a follow-up period of 3-7 years [2-4, 7], revisions to ankle arthrodeses will be necessary in $10-15 \%$ of all cases.

Conflict of interest None.

\section{References}

1. Whittle AP. Malunited fractures. In: Campbell's operative orthopaedics. Philadelphia: Mosby Elsevier; 2008. p. 3461-3525.

2. Weber D, Friederich NF, Müller W. Lengthening osteotomy of the fibula for post-traumatic malunion. Int Orthop. 1998;22: $149-52$. image. Right ankle, lateral view. Oblique osteotomy. DCP plate (2.7 mm, AO-ASIF). e, f. Radiographs 10 days after correction osteotomy. Reduction of the talus. Osteosynthesis of medial and lateral malleolus

3. Sinha A, Sirikonda S, Giotakis N. Fibular lengthening for malunited ankle fractures. Foot Ankle Int. 2008;29:1136-40.

4. Marti RK, Raaymakers EL, Nolte PA. Malunited ankle fractures: the late results of reconstruction. J Bone Joint Surg. 1990;72B: 709-13.

5. Yablon IG, Leach RE. Reconstruction of malunited fractures of the lateral malleolus. J Bone Joint Surg. 1989;71A:521-7.

6. Hughes JL, Weber H, Willenegger H, Kuner EH. Evaluation of ankle fractures: non-operative and operative treatment. Clin Orthop. 1979;138:111-9.

7. Offierski CM, Graham JD, Hall JH, Harris WR, Schatzker JL. Late revision of fibular malunion in ankle fractures. Clin Orthop. 1982;171:145-9.

8. Weber BG, Simpson LA. Corrective lengthening osteotomy of the fibula. Clin Orthop. 1985;199:61-7.

9. Weber M. Trimalleolar fractures with impaction of the posteromedial tibial plafond: implications for talar stability. Foot Ankle Int. 2004;25:716-27.

10. Weber M, Ganz R. Malunion following trimalleolar fracture with posterolateral subluxation of the talus-reconstruction including the posterior malleolus. Foot Ankle Int. 2003;24:338-44.

11. Harstall R, Lehmann O, Krause F, Weber M. Supramalleolar lateral closing wedge osteotomy for the treatment of varus ankle arthrosis. Foot Ankle Int. 2007;28:542-8.

12. Tencer AF. Mechanisms of internal fixation of bone. In: An YH, editors. Internal fixation in osteoporotic bone. New York: Thieme; 2002. p. 40-50.

13. Hertel R, Jost B. Basic principles and techniques of internal fixation in osteoporotic bone. In: An YH, editors. Internal fixation in osteoporotic bone. New York: Thieme; 2002. p. 108-115. 